\title{
Dynamical Effects in the Study of Supported Nanocrystals using Electron Holography
}

\author{
R.E. Dunin-Borkowski, C.B. Boothroyd and M. Beleggia \\ Center for Electron Nanoscopy, Technical University of Denmark, DK-2800 Lyngby, \\ Denmark
}

Off-axis electron holography is a transmission electron microscopy technique that can be used to record the phase shift of the electron wave that has passed through a thin specimen [1]. The phase shift is sensitive to both the electrostatic potential and the magnetic induction in the specimen. The technique is now widely used to measure electrostatic potentials in semiconductor devices and magnetic fields in nanocrystals and thin films. Here, we discuss the application of electron holography to the characterization of electrostatic potentials in supported metal particles, which are of interest for applications in heterogeneous catalysis.

Measurements of mean inner potentials are inherently sensitive to the structural and chemical states of materials, as well as to charge transfer. Recently, several intriguing reports have suggested that the mean inner potentials of sub-10-nm metal nanocrystals, measured using electron holography, may be substantially higher than those measured from larger crystals [24]. These observations appear to indicate that electron holography can be used to provide useful information about the catalytic properties of supported metal particles. However, many factors may influence such measurements, including changes in lattice parameter, the presence of adsorbates on the particle surfaces [5], the effect of dynamical diffraction on the measured phase shift, the accuracy and precision with which the particle thickness in the electron beam direction can be measured, and charging of the particles and their supports as a result of electron beam irradiation.

Here, in order to illustrate one of the factors that may affect measurements of the mean inner potentials of supported nanoparticles, Figs 1 and 2 show the dramatic influence of dynamical diffraction on the phase shifts of $\mathrm{Au}$ and graphite, in the form of multislice simulations of the phase shift of the 000 beam. Figure 1 shows phase shifts plotted as a function of specimen thickness for several zone axis orientations for each material. For $\mathrm{Au}$, the calculations show that the phase shift can be more than $50 \%$ lower than kinematic predictions at a specimen thickness of only $2 \mathrm{~nm}$. For graphite, the calculated phase shifts can be close to zero or even negative for zone axes that lie in the plane of the graphene layers. The plots show that, for certain values of specimen thickness and specimen tilt, phase shifts for graphite can be larger than those for $\mathrm{Au}$. Figure 2 shows corresponding calculations of phase shifts plotted as a function of specimen tilt angle from $\mathrm{Au}$ [011] and graphite [001], for a specimen thickness of $100 \mathrm{~nm}$. The black and white levels in the figures correspond to effective values of mean inner potential (i.e., the values that would be inferred from the phase shift if the specimen thickness were known accurately) of between $-3.8 \mathrm{~V}$ and $64.6 \mathrm{~V}$ for $\mathrm{Au}$, and between $4.8 \mathrm{~V}$ and $22.8 \mathrm{~V}$ for graphite. These changes with specimen tilt (and specimen thickness) can be compared with the single values of $29.8 \mathrm{~V}$ and $13.5 \mathrm{~V}$ that would be predicted for the mean inner potentials of Au and graphite, respectively, in the absence of dynamical diffraction [6].

The simulations in Figs 1 and 2 suggest that the effects of dynamical diffraction will complicate attempts to relate holographic measurements of mean inner potentials of metal particles to parameters of interest for catalysis, such as the physical or electronic state of the surface of the nanocrystal or charge transfer between the particle and its support. 


\section{References}

[1] R.E. Dunin-Borkowski et al., Encyclopedia of Nanoscience and Nanotechnology, American Scientific Publishers: Stevenson Ranch, CA, Vol. 3 (2004) 41.

[2] K. Okazaki et al., Appl. Catal. A 291 (2005) 45.

[3] R. Popescu et al., Phys. Rev. B 76 (2007) 235411.

[4] L. Ortolani et al., J. Eur. Ceram. Soc. 27 (2007) 4131.

[5] R.M. Wang et al., Phys. Rev. Lett. 100 (2008) 017205.

[6] Discussions with T. Kasama, T.W. Hansen, J.B. Wagner, R.S. Pennington and J.R. Jinschek are gratefully acknowledged.
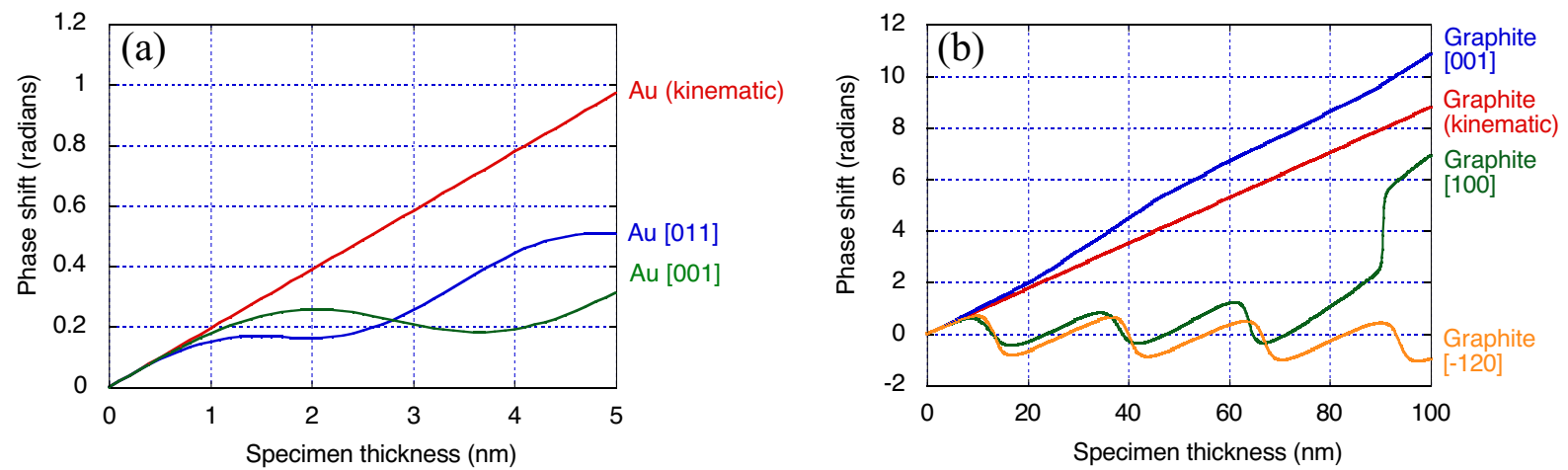

FIG. 1. Dynamical calculations of phase shifts of the 000 beam plotted for the indicated zone axis orientations of (a) $\mathrm{Au}$ and (b) graphite, for an accelerating voltage of $300 \mathrm{kV}$. Each calculation is performed using a multislice calculation with a single projected potential created using neutral atom scattering factors and no absorption. Kinematic predictions are also shown. Note the different specimen thickness ranges and vertical axes in the two graphs.
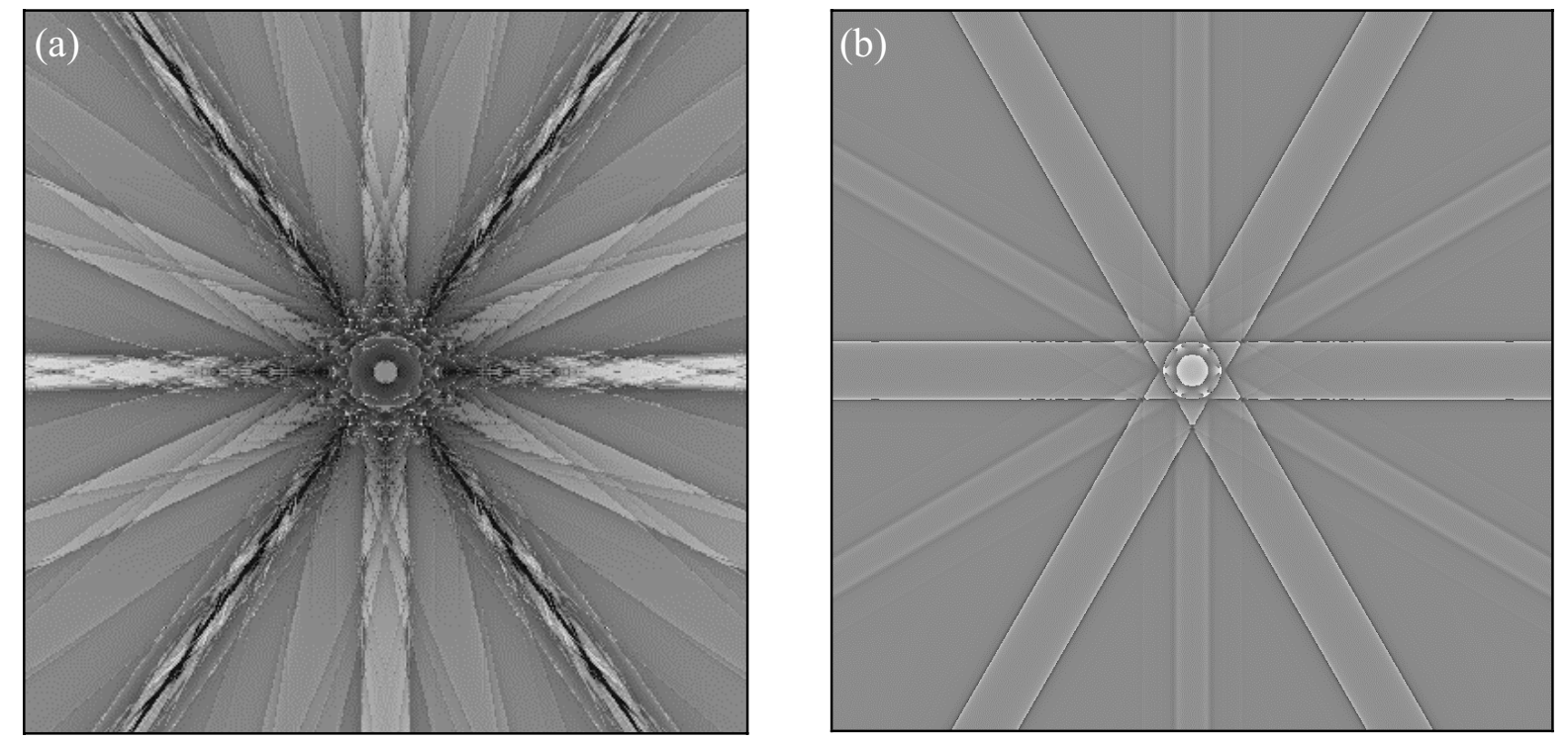

FIG. 2. Dynamical calculations of phase shifts of the 000 beam plotted as a function of specimen tilt angle up to $\pm 100 \mathrm{mrad}$ horizontally and $\pm 100 \mathrm{mrad}$ vertically at a single specimen thickness of $100 \mathrm{~nm}$, starting from (a) $\mathrm{Au}$ [011] and (b) graphite [001]. If these phase values were measured experimentally and the specimen thickness were known, then the black and white levels in the figures would correspond to inferred mean inner potentials of (a) $-3.8 \mathrm{~V}$ and $64.6 \mathrm{~V}$ and (b) $4.8 \mathrm{~V}$ and $22.8 \mathrm{~V}$. (In the absence of dynamical diffraction, the mean inner potentials of $\mathrm{Au}$ and graphite are calculated to be $29.8 \mathrm{~V}$ and $13.5 \mathrm{~V}$, respectively). 